\title{
Fast Crash Recovery in RAMCloud
}

\author{
Diego Ongaro, Stephen M. Rumble, Ryan Stutsman, \\ John Ousterhout, and Mendel Rosenblum
}

\author{
Stanford University
}

\begin{abstract}
RAMCloud is a DRAM-based storage system that provides inexpensive durability and availability by recovering quickly after crashes, rather than storing replicas in DRAM. RAMCloud scatters backup data across hundreds or thousands of disks, and it harnesses hundreds of servers in parallel to reconstruct lost data. The system uses a log-structured approach for all its data, in DRAM as well as on disk; this provides high performance both during normal operation and during recovery. RAMCloud employs randomized techniques to manage the system in a scalable and decentralized fashion. In a 60-node cluster, RAMCloud recovers $35 \mathrm{~GB}$ of data from a failed server in 1.6 seconds. Our measurements suggest that the approach will scale to recover larger memory sizes (64 GB or more) in less time with larger clusters.
\end{abstract}

\section{Categories and Subject Descriptors}

D.4.7 [Operating Systems]: Organization and Design—Distributed systems; D.4.2 [Operating Systems]: Storage Management_Main memory; D.4.5 [Operating Systems]: Reliability_Fault-tolerance; D.4.8 [Operating Systems]: Performance-Measurements

\section{General Terms}

Design, Measurement, Performance, Reliability, Experimentation

\section{Keywords}

Storage systems, Main memory databases, Crash recovery, Scalability

\section{INTRODUCTION}

The role of DRAM in storage systems has been increasing rapidly in recent years, driven by the needs of large-scale Web applications. These applications manipulate very large datasets with an intensity that cannot be satisfied by disks alone. As a result, applications are keeping more and more of their data in DRAM. For example, large-scale caching systems such as memcached [3] are being widely used (in 2009 Facebook used a total of 150 TB of DRAM in memcached and other caches for a

Permission to make digital or hard copies of all or part of this work for personal or classroom use is granted without fee provided that copies are not made or distributed for profit or commercial advantage and that copies bear this notice and the full citation on the first page. To copy otherwise, to republish, to post on servers or to redistribute to lists, requires prior specific permission and/or a fee. 
database containing 200 TB of disk storage [15]), and the major Web search engines now keep their search indexes entirely in DRAM.

Although DRAM's role is increasing, it still tends to be used in limited or specialized ways. In most cases DRAM is just a cache for some other storage system such as a database; in other cases (such as search indexes) DRAM is managed in an application-specific fashion. It is difficult for developers to use DRAM effectively in their applications; for example, the application must manage consistency between caches and the backing storage. In addition, cache misses and backing store overheads make it difficult to capture DRAM's full performance potential.

RAMCloud is a general-purpose storage system that makes it easy for developers to harness the full performance potential of large-scale DRAM storage. It keeps all data in DRAM all the time, so there are no cache misses. RAMCloud storage is durable and available, so developers need not manage a separate backing store. RAMCloud is designed to scale to thousands of servers and hundreds of terabytes of data while providing uniform low-latency access (5-10 $\mu$ s round-trip times for small read operations).

The most important factor in the design of RAMCloud was the need to provide a high level of durability and availability without impacting system performance. Replicating all data in DRAM would have solved some availability issues, but with $3 \mathrm{x}$ replication this would have tripled the cost and energy usage of the system. Instead, RAMCloud keeps only a single copy of data in DRAM; redundant copies are kept on disk or flash, which is both cheaper and more durable than DRAM. However, this means that a server crash will leave some of the system's data unavailable until it can be reconstructed from secondary storage.

RAMCloud's solution to the availability problem is fast crash recovery: the system reconstructs the entire contents of a lost server's memory (64 GB or more) from disk and resumes full service in 1-2 seconds. We believe this is fast enough to be considered "continuous availability" for most applications.

This paper describes and evaluates RAMCloud's approach to fast recovery. There are several interesting aspects to the RAMCloud architecture:

- Harnessing scale: RAMCloud takes advantage of the system's large scale to recover quickly after crashes. Each server scatters its backup data across all of the other servers, allowing thousands of disks to participate in recovery. Hundreds of recovery masters work together to avoid network and CPU bottlenecks while recovering data. RAMCloud uses both data parallelism and pipelining to speed up recovery.

- Log-structured storage: RAMCloud uses techniques similar to those from log-structured file systems [21], not just for information on disk but also for information in DRAM. The logstructured approach provides high performance and simplifies many issues related to crash recovery.

- Randomization: RAMCloud uses randomized approaches to make decisions in a distributed and scalable fashion. In some cases randomization is combined with refinement: a server selects several candidates at random and then chooses among them using more detailed information; this provides near-optimal results at low cost.

- Tablet profiling: RAMCloud uses a novel dynamic tree structure to track the distribution of data within tables; this helps divide a server's data into partitions for fast recovery.

We have implemented the RAMCloud architecture in a working system and evaluated its crash recovery properties. Our 60-node cluster recovers in 1.6 seconds from the failure of a server with 35 GB of data, and the approach scales so that larger clusters can recover larger memory sizes in less time. 
Measurements of our randomized replica placement algorithm show that it produces uniform allocations that minimize recovery time and that it largely eliminates straggler effects caused by varying disk speeds.

Overall, fast crash recovery allows RAMCloud to provide durable and available DRAM-based storage for the same price and energy usage as today's volatile DRAM caches.

\section{RAMCLOUD}

Crash recovery and normal request processing are tightly intertwined in RAMCloud, so this section provides background on the RAMCloud concept and the basic data structures used to process requests. We have omitted some details because of space limitations.

\subsection{Basics}

RAMCloud is a storage system where every byte of data is present in DRAM at all times. The hardware for RAMCloud consists of hundreds or thousands of off-the-shelf servers in a single datacenter, each with as much DRAM as is cost-effective (24 to 64 GB today). RAMCloud aggregates the DRAM of all these servers into a single coherent storage system. It uses backup copies on disk or flash to make its storage durable and available, but the performance of the system is determined by DRAM, not disk.

The RAMCloud architecture combines two interesting properties: low latency and large scale. First, RAMCloud is designed to provide the lowest possible latency for remote access by applications in the same datacenter. Our goal is end-to-end times of 5-10 $\mu$ s for reading small objects in datacenters with tens of thousands of machines. This represents an improvement of 50-5,000x over existing datacenter-scale storage systems.

Unfortunately, today's datacenters cannot meet RAMCloud's latency goals (Ethernet switches and NICs typically add at least 200-500 $\mu$ s to round-trip latency in a large datacenter). Thus we use lowlatency Infiniband NICs and switches in our development environment as an approximation to the networking hardware we hope will be commonplace in a few years; this makes it easier to explore latency issues in the RAMCloud software. The current RAMCloud system supports $5 \mu$ s reads in a small cluster, and each storage server can handle about 1 million small read requests per second.

The second important property of RAMCloud is scale: a single RAMCloud cluster must support thousands of servers in order to provide a coherent source of data for large applications. Scale creates several challenges, such as the likelihood of frequent component failures and the need for distributed decision-making to avoid bottlenecks. However, scale also creates opportunities, such as the ability to enlist large numbers of resources on problems like fast crash recovery.

RAMCloud's overall goal is to enable a new class of applications that manipulate large datasets more intensively than has ever been possible. For more details on the motivation for RAMCloud and some of its architectural choices, see [18].

\subsection{Data Model}

The current data model in RAMCloud is a simple key-value store. RAMCloud supports any number of tables, each of which contains any number of objects. An object consists of a 64-bit identifier, a variable-length byte array (up to $1 \mathrm{MB}$ ), and a 64-bit version number. RAMCloud provides a simple set of operations for creating and deleting tables and for reading, writing, and deleting objects within a table. Objects are addressed with their identifiers and are read and written in their entirety. There is no built-in support for atomic updates to multiple objects, but RAMCloud does provide a conditional update ("replace the contents of object $O$ in table $T$ only if its current version number is $V$ "), which can be used to implement more complex transactions in application software. In the future we plan to experiment with more powerful features such as indexes, mini-transactions [4], and support for large graphs. 


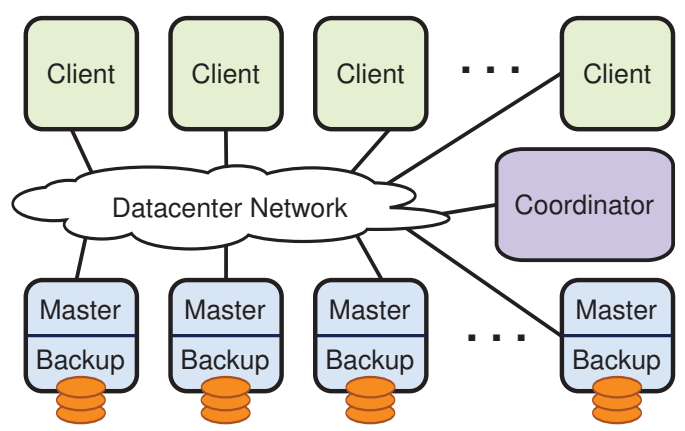

Figure 1: RAMCloud cluster architecture. Each storage server contains a master and a backup. A central coordinator manages the server pool and tablet configuration. Client applications run on separate machines and access RAMCloud using a client library that makes remote procedure calls.

\subsection{System Structure}

As shown in Figure 1, a RAMCloud cluster consists of a large number of storage servers, each of which has two components: a master, which manages RAMCloud objects in its DRAM and services client requests, and a backup, which stores redundant copies of objects from other masters using its disk or flash memory. Each RAMCloud cluster also contains one distinguished server called the coordinator. The coordinator manages configuration information such as the network addresses of the storage servers and the locations of objects; it is not involved in most client requests.

The coordinator assigns objects to storage servers in units of tablets: consecutive key ranges within a single table. Small tables are stored in their entirety on a single storage server; larger tables are split across multiple servers. Client applications do not have control over the tablet configuration; however, they can achieve some locality by taking advantage of the fact that small tables (and adjacent keys in large tables) are stored together on a single server.

The coordinator stores the mapping between tablets and storage servers. The RAMCloud client library maintains a cache of this information, fetching the mappings for each table the first time it is accessed. Clients can usually issue storage requests directly to the relevant storage server without involving the coordinator. If a client's cached configuration information becomes stale because a tablet has moved, the client library discovers this when it makes a request to a server that no longer contains the tablet, at which point it flushes the stale data from its cache and fetches up-to-date information from the coordinator. Clients use the same mechanism during crash recovery to find the new location for data.

\subsection{Managing Replicas}

The internal structure of a RAMCloud storage server is determined primarily by the need to provide durability and availability. In the absence of these requirements, a master would consist of little more than a hash table that maps from 〈table identifier, object identifier $\rangle$ pairs to objects in DRAM. The main challenge is providing durability and availability without sacrificing performance or greatly increasing system cost.

One possible approach to availability is to replicate each object in the memories of several servers. However, with a typical replication factor of three, this approach would triple both the cost and energy usage of the system (each server is already fully loaded, so adding more memory would also require adding more servers and networking). The cost of main-memory replication can be reduced by using coding techniques such as parity striping [20], but this makes crash recovery considerably more expensive. Furthermore, DRAM-based replicas are still vulnerable in the event of power failures.

Instead, RAMCloud keeps only a single copy of each object in DRAM, with redundant copies on 


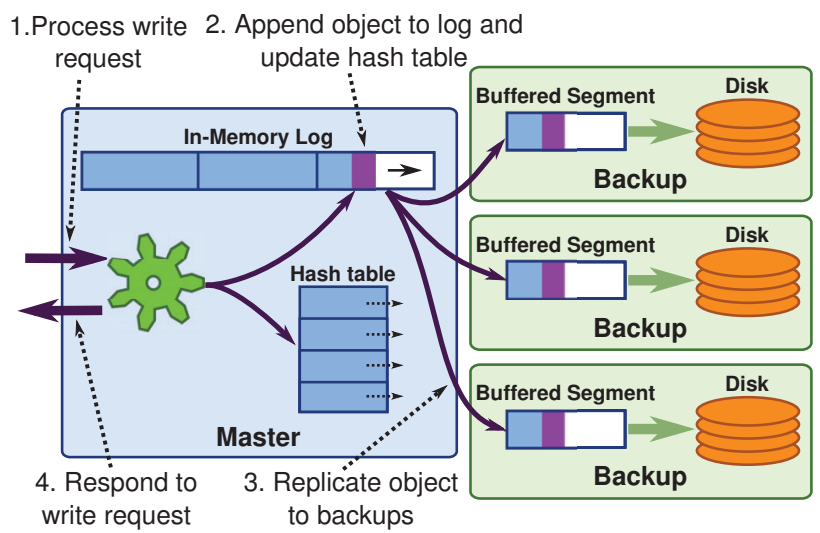

Figure 2: When a master receives a write request, it updates its in-memory log and forwards the new data to several backups, which buffer the data in their memory. The data is eventually written to disk or flash in large batches. Backups must use an auxiliary power source to ensure that buffers can be written to stable storage after a power failure.

secondary storage such as disk or flash. This makes replication nearly free in terms of cost and energy usage (the DRAM for primary copies will dominate both of these factors), but it raises two issues. First, the use of slower storage for backup might impact the normal-case performance of the system (e.g., by waiting for synchronous disk writes). Second, this approach could result in long periods of unavailability or poor performance after server crashes, since the data will have to be reconstructed from secondary storage. Section 2.5 describes how RAMCloud solves the performance problem, and Section 3 deals with crash recovery.

\subsection{Log-Structured Storage}

RAMCloud manages object data using a logging approach. This was originally motivated by the desire to transfer backup data to disk or flash as efficiently as possible, but it also provides an efficient memory management mechanism, enables fast recovery, and has a simple implementation. The data for each master is organized as a $\log$ as shown in Figure 2. When a master receives a write request, it appends the new object to its in-memory log and forwards that log entry to several backup servers. The backups buffer this information in memory and return immediately to the master without writing to disk or flash. The master completes its request and returns to the client once all of the backups have acknowledged receipt of the log data. When a backup's buffer fills, it writes the accumulated $\log$ data to disk or flash in a single large transfer, then deletes the buffered data from its memory.

Backups must ensure that buffered log data is as durable as data on disk or flash (i.e., information must not be lost in a power failure). One solution is to use new DIMM memory modules that incorporate flash memory and a super-capacitor that provides enough power for the DIMM to write its contents to flash after a power outage [2]; each backup could use one of these modules to hold all of its buffered log data. Other alternatives are per-server battery backups that extend power long enough for RAMCloud to flush buffers, or enterprise disk controllers with persistent cache memory.

RAMCloud manages its logs using techniques similar to those in log-structured file systems [21]. Each master's log is divided into $8 \mathrm{MB}$ segments. The master keeps a count of unused space within each segment, which accumulates as objects are deleted or overwritten. It reclaims wasted space by occasionally invoking a log cleaner; the cleaner selects one or more segments to clean, reads the live records from the segments and rewrites them at the head of the log, then deletes the cleaned segments along with their backup copies. Segments are also the unit of buffering and I/O on backups; the large segment size enables efficient I/O for both disk and flash. 


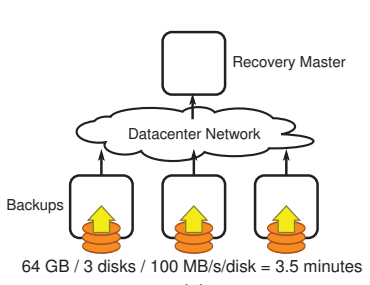

(a)

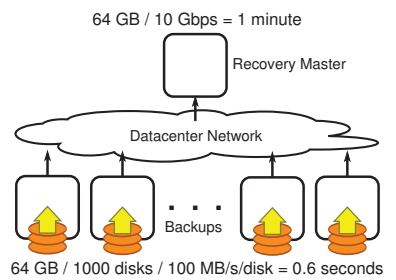

(b)

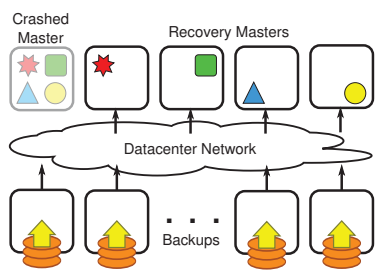

(c)

Figure 3: (a) Disk bandwidth is a recovery bottleneck if each master's data is mirrored on a small number of backup machines. (b) Scattering log segments across many backups removes the disk bottleneck, but recovering all data on one recovery master is limited by the network interface and CPU of that machine. (c) Fast recovery is achieved by partitioning the data of the crashed master and recovering each partition on a separate recovery master.

RAMCloud uses a log-structured approach not only for backup storage, but also for information in DRAM: the memory of a master is structured as a collection of log segments identical to those stored on backups. This allows masters to manage both their in-memory data and their backup data using a single mechanism. The log provides an efficient memory management mechanism, with the cleaner implementing a form of generational garbage collection. In order to support random access to objects in memory, each master keeps a hash table that maps from 〈table identifier, object identifier pairs to the current version of an object in a segment. The hash table is used both to look up objects during storage operations and to determine whether a particular object version is the current one during cleaning (for example, if there is no hash table entry for a particular object in a segment being cleaned, it means the object has been deleted).

The buffered logging approach allows writes to complete without waiting for disk operations, but it limits overall system throughput to the bandwidth of the backup storage. For example, each RAMCloud server can handle about 300,000 100-byte writes/second (versus 1 million reads/second) assuming 2 disks per server, $100 \mathrm{MB} / \mathrm{s}$ write bandwidth for each disk, 3 disk replicas of each object, and a 100\% bandwidth overhead for log cleaning. Additional disks can be used to boost write throughput.

\section{RECOVERY}

When a RAMCloud storage server crashes, the objects that had been present in its DRAM must be reconstructed by replaying its log. This requires reading log segments from backup storage, processing the records in those segments to identify the current version of each live object, and reconstructing the hash table used for storage operations. The crashed master's data will be unavailable until the hash table has been reconstructed.

Fortunately, if the period of unavailability can be made very short, so that it is no longer than other delays that are common in normal operation, and if crashes happen infrequently, then crash recovery will be unnoticeable to the application's users. We believe that 1-2 second recovery is fast enough to constitute "continuous availability" for most applications; our goal is to achieve this speed for servers with at least $64 \mathrm{~GB}$ of memory.

\subsection{Using Scale}

The key to fast recovery in RAMCloud is to take advantage of the massive resources of the cluster. This subsection introduces RAMCloud's overall approach for harnessing scale; the following subsections describe individual elements of the mechanism in detail.

As a baseline, Figure 3a shows a simple mirrored approach where each master chooses 3 backups and stores copies of all its log segments on each backup. Unfortunately, this creates a bottleneck for recovery because the master's data must be read from only a few disks. In the configuration of Figure $3 \mathrm{a}$ with 3 disks, it would take about 3.5 minutes to read $64 \mathrm{~GB}$ of data. 
RAMCloud works around the disk bottleneck by using more disks during recovery. Each master scatters its log data across all of the backups in the cluster (each segment on a different set of backups) as shown in Figure 3b. During recovery, these scattered log segments can be read simultaneously; with 1,000 disks, 64 GB of data can be read into memory in less than one second.

Once the segments have been read from disk into backups' memories, they must be combined to find the most recent version for each object (no backup can tell in isolation whether a particular object in a particular segment is the most recent version). One approach is to send all the log segments to a single recovery master and replay the log on that master, as in Figure $3 \mathrm{~b}$. Unfortunately, the recovery master is a bottleneck in this approach: with a 10 Gbps network interface, it will take about 1 minute to read 64 GB of data, and the master's CPU will also be a bottleneck.

To eliminate the recovery master as the bottleneck, RAMCloud uses multiple recovery masters as shown in Figure 3c. During recovery RAMCloud divides the objects of the crashed master into partitions of roughly equal size. Each partition is assigned to a different recovery master, which fetches the log data for the partition's objects from backups and incorporates those objects into its own log and hash table. With 100 recovery masters operating in parallel, 64 GB of data can be transferred over a 10 Gbps network in less than 1 second. As will be shown in Section 4, this is also enough time for each recovery master's CPU to process the incoming data.

Thus, the overall approach to recovery in RAMCloud is to combine the disk bandwidth, network bandwidth, and CPU cycles of thousands of backups and hundreds of recovery masters. The subsections below describe how RAMCloud divides its work among all of these resources and how it coordinates the resources to recover in 1-2 seconds.

\subsection{Scattering Log Segments}

For fastest recovery the log segments for each RAMCloud master should be distributed uniformly across all of the backups in the cluster. However, there are several factors that complicate this approach:

- Segment placement must reflect failure modes. For example, a segment's master and each of its backups must reside in different racks, in order to protect against top-of-rack switch failures and other problems that disable an entire rack.

- Different backups may have different bandwidth for I/O (different numbers of disks, different disk speeds, or different storage classes such as flash memory); segments should be distributed so that each backup uses the same amount of time to read its share of the data during recovery.

- All of the masters are writing segments simultaneously; they should coordinate to avoid overloading any individual backup. Backups have limited buffer space.

- Storage servers are continuously entering and leaving the cluster, which changes the pool of available backups and may unbalance the distribution of segments.

Making decisions such as segment replica placement in a centralized fashion on the coordinator would limit RAMCloud's scalability. For example, a cluster with 10,000 servers could back up 100,000 or more segments per second; this could easily cause the coordinator to become a performance bottleneck.

Instead, each RAMCloud master decides independently where to place each replica, using a combination of randomization and refinement. When a master needs to select a backup for a segment, it chooses several candidates at random from a list of all backups in the cluster. Then it selects the best candidate, using its knowledge of where it has already allocated segment replicas and information about the speed of each backup's disk (backups measure the speed of their disks when they start up 
and provide this information to the coordinator, which relays it on to masters). The best backup is the one that can read its share of the master's segment replicas most quickly from disk during recovery. A backup is rejected if it is in the same rack as the master or any other replica for the current segment. Once a backup has been selected, the master contacts that backup to reserve space for the segment. At this point the backup can reject the request if it is overloaded, in which case the master selects another candidate.

The use of randomization eliminates pathological behaviors such as all masters choosing the same backups in a lock-step fashion. Adding the refinement step provides a solution nearly as optimal as a centralized manager (see [17] and [5] for a theoretical analysis). For example, if a master scatters 8,000 segments across 1,000 backups using a purely random approach, backups will have 8 segments on average. However, some backups are likely to end up with 15-20 segments, which will result in uneven disk utilization during recovery. Adding just a small amount of choice makes the segment distribution nearly uniform and also allows for compensation based on other factors such as disk speed (see Section 4.4). This mechanism also handles the entry of new backups gracefully: a new backup is likely to be selected more frequently than existing backups until every master has taken full advantage of it.

RAMCloud masters mark one of the replicas for each segment as the primary replica. Only the primary replicas are read during recovery (unless they are unavailable), and the performance optimizations described above consider only primary replicas.

We considered the possibility of storing one of the backup replicas on the same machine as the master. This would reduce network bandwidth requirements, but it has two disadvantages. First, it would reduce system fault tolerance: the master already has one copy in its memory, so placing a second copy on the master's disk provides little benefit. If the master crashes, the disk copy will be lost along with the memory copy; it would only provide value in a cold start after a power failure. Second, storing one replica on the master would limit the burst write bandwidth of a master to the bandwidth of its local disks. In contrast, with all replicas scattered, a single master can potentially use the disk bandwidth of the entire cluster (up to the limit of its network interface).

\subsection{Failure Detection}

RAMCloud detects server failures in two ways. First, RAMCloud clients will notice if a server fails to respond to a remote procedure call. Second, RAMCloud checks its own servers to detect failures even in the absence of client activity; this allows RAMCloud to replace lost replicas before multiple crashes cause permanent data loss. Each RAMCloud server periodically issues a ping RPC to another server chosen at random and reports failures to the coordinator. This is another example of using a randomized distributed approach in place of a centralized approach. The probability of detecting a crashed machine in a single round of pings is about $63 \%$ for clusters with 100 or more nodes; the odds are greater than $99 \%$ that a failed server will be detected within five rounds.

In either case, server failures are reported to the coordinator. The coordinator verifies the problem by attempting to communicate with the server itself, then initiates recovery if the server does not respond. Timeouts must be relatively short (tens of milliseconds) so that they don't significantly delay recovery. See Section 5 for a discussion of the risks introduced by short timeouts.

\subsection{Recovery Flow}

The coordinator supervises the recovery process, which proceeds in three phases:

1. Setup. The coordinator finds all replicas of all log segments belonging to the crashed master, selects recovery masters, and assigns each recovery master a partition to recover.

2. Replay. Recovery masters fetch log segments in parallel and incorporate the crashed master's partitions into their own logs. 
3. Cleanup. Recovery masters begin serving requests, and the crashed master's log segments are freed from backup storage.

These phases are described in more detail below.

\subsection{Setup}

\subsubsection{Finding Log Segment Replicas}

At the start of recovery, replicas of the crashed master's segments must be located among the cluster's backups. RAMCloud does not keep a centralized map of replicas since it would be difficult to scale and would hinder common-case performance. Only masters know where their segments are replicated, but this information is lost when they crash.

The coordinator reconstructs the locations of the crashed master's replicas by querying all of the backups in the cluster. Each backup responds with a list of the replicas it has stored for the crashed master (backups maintain this index in memory). The coordinator then aggregates the responses into a single location map. By using RAMCloud's fast RPC system and querying multiple backups in parallel, the segment location information is collected quickly.

\subsubsection{Detecting Incomplete Logs}

After backups return their lists of replicas, the coordinator must determine whether the reported segment replicas form the entire log of the crashed master. The redundancy in RAMCloud makes it highly likely that the entire log will be available, but the system must be able to detect situations where some data is missing (such as network partitions).

RAMCloud avoids centrally tracking the list of the segments that comprise a master's log by making each log self-describing; the completeness of the log can be verified using data in the log itself. Each segment includes a log digest, which is a list of identifiers for all segments in the log at the time this segment was written. Log digests are small (less than $1 \%$ storage overhead even when uncompressed, assuming $8 \mathrm{MB}$ segments and 8,000 segments per master).

This leaves a chance that all the replicas for the newest segment in the log are unavailable, in which case the coordinator would not be able to detect that the log is incomplete (the most recent digest it could find would not list the newest segment). To prevent this, when a master creates a new segment replica it makes its transition to the new digest carefully. First, a new digest is inserted in the new replica, and it is marked as active. Then, after the new active digest is durable, a final update to the prior active digest marks it as inactive. This ordering ensures the log always has an active digest, even if the master crashes between segments. Two active log digests may be discovered during recovery, but the coordinator simply ignores the newer one since its segment must be empty.

If the active log digest and a replica for each segment cannot be found, then RAMCloud cannot recover the crashed master. In this unlikely case, RAMCloud notifies the operator and waits for backups to return to the cluster with replicas for each of the missing segments. Alternatively, at the operator's discretion, RAMCloud can continue recovery with loss of data.

\subsubsection{Starting Partition Recoveries}

Next, the coordinator must divide up the work of recovering the crashed master. The choice of partitions for a crashed master is made by the master itself: during normal operation each master analyzes its own data and computes a set of partitions that would evenly divide the work of recovery. This information is called a will (it describes how a master's assets should be divided in the event of its demise). Masters periodically upload their wills to the coordinator. Section 3.9 describes how masters compute their wills efficiently.

During recovery setup, the coordinator assigns each of the partitions in the crashed master's will to an existing master within the cluster. Each of these recovery masters receives two things from the 


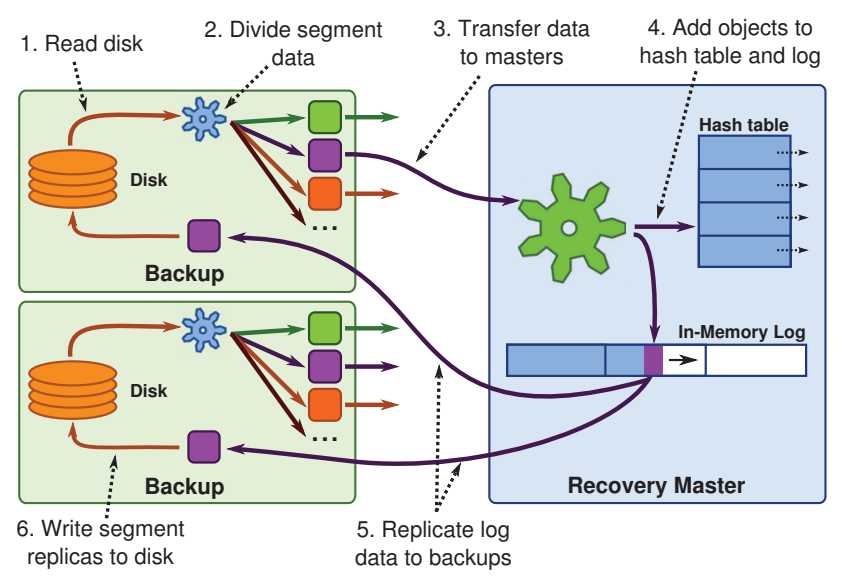

Figure 4: During recovery, segment data flows from disk or flash on a backup over the network to a recovery master, then back to new backups as part of the recovery master's log.

coordinator: a list of the locations of all the crashed master's log segments and a list of tablets that the recovery master must recover and incorporate into the data it manages.

\subsection{Replay}

The vast majority of recovery time is spent replaying segments to reconstruct partitions on the recovery masters. During replay the contents of each segment are processed in six stages (see Figure 4):

1. The segment is read from disk into the memory of a backup.

2. The backup divides the records in the segment into separate groups for each partition based on table and object identifiers in the log records.

3. The records for each partition are transferred over the network to the recovery master for that partition.

4. The recovery master incorporates the data into its in-memory log and hash table.

5. As the recovery master fills segments in memory, it replicates those segments over the network to backups with the same scattering mechanism used in normal operation.

6. The backups write the new segment replicas to disk or flash.

RAMCloud harnesses concurrency in two dimensions during recovery. The first dimension is data parallelism: different backups read different segments from disk in parallel, different recovery masters reconstruct different partitions in parallel, and so on. The second dimension is pipelining: all of the six stages listed above proceed in parallel, with a segment as the basic unit of work. While one segment is being read from disk on a backup, another segment is being partitioned by that backup's $\mathrm{CPU}$, and records from another segment are being transferred to a recovery master; similar pipelining occurs on recovery masters. For fastest recovery all of the resources of the cluster should be kept fully utilized, including disks, CPUs, and the network.

\subsection{Segment Replay Order}

In order to maximize concurrency, recovery masters and backups operate independently. As soon as the coordinator contacts each backup to obtain its list of segments, the backup begins prefetching segments from disk and dividing them by partition. At the same time, masters fetch segment data 
from backups and replay it. Ideally backups will constantly run ahead of masters, so that segment data is ready and waiting whenever a recovery master requests it. However, this only works if the recovery masters and backups process segments in the same order. If a recovery master accidentally requests the last segment in the backup's order then the master will stall: it will not receive any data to process until the backup has read all of its segments.

In order to avoid pipeline stalls, each backup decides in advance the order in which it will read its segments. It returns this information to the coordinator during the setup phase, and the coordinator includes the order information when it communicates with recovery masters to initiate recovery. Each recovery master uses its knowledge of backup disk speeds to estimate when each segment's data is likely to be loaded. It then requests segment data in order of expected availability. (This approach causes all masters to request segments in the same order; we could introduce randomization to avoid contention caused by lock-step behavior.)

Unfortunately, there will still be variations in the speed at which backups read and process segments. In order to avoid stalls because of slow backups, each master keeps several concurrent requests for segment data outstanding at any given time during recovery; it replays segment data in the order that the requests return.

Because of the optimizations described above, recovery masters will end up replaying segments in a different order than the one in which the segments were originally written. Fortunately, the version numbers in log records allow the log to be replayed in any order without affecting the result. During replay each master simply retains the version of each object with the highest version number, discarding any older versions that it encounters.

Although each segment has multiple replicas stored on different backups, only the primary replicas are read during recovery; reading more than one would waste valuable disk bandwidth. Masters identify primary replicas when scattering their segments as described in Section 3.2. During recovery each backup reports all of its segments, but it identifies the primary replicas and only prefetches the primary replicas from disk. Recovery masters request non-primary replicas only if there is a failure reading the primary replica.

\subsection{Cleanup}

After a recovery master completes the recovery of its assigned partition, it notifies the coordinator that it is ready to service requests. The coordinator updates its configuration information to indicate that the master now owns the tablets in the recovered partition, at which point the partition is available for client requests. Clients with failed RPCs to the crashed master have been waiting for new configuration information to appear; they discover it and retry their RPCs with the new master. Recovery masters can begin service independently without waiting for other recovery masters to finish.

Once all recovery masters have completed recovery, the coordinator contacts each of the backups again. At this point the backups free the storage for the crashed master's segments, since it is no longer needed. Recovery is complete once all of the backups have been notified.

\subsection{Tablet Profiling}

Each master is responsible for creating a will, which describes how its objects should be partitioned during recovery. A partition consists of one or more tablets. The master should balance its partitions so that they require roughly equal time to recover, and the partitions should be sized based on the desired recovery time. The master's storage is not actually partitioned during normal operation as this would create unnecessary overheads; partitioning only occurs during recovery. The master uploads its will to the coordinator and updates the will as its data evolves.

RAMCloud computes wills using tablet profiles. Each tablet profile tracks the distribution of resource usage within a single table or tablet in a master. It consists of a collection of buckets, each of which 


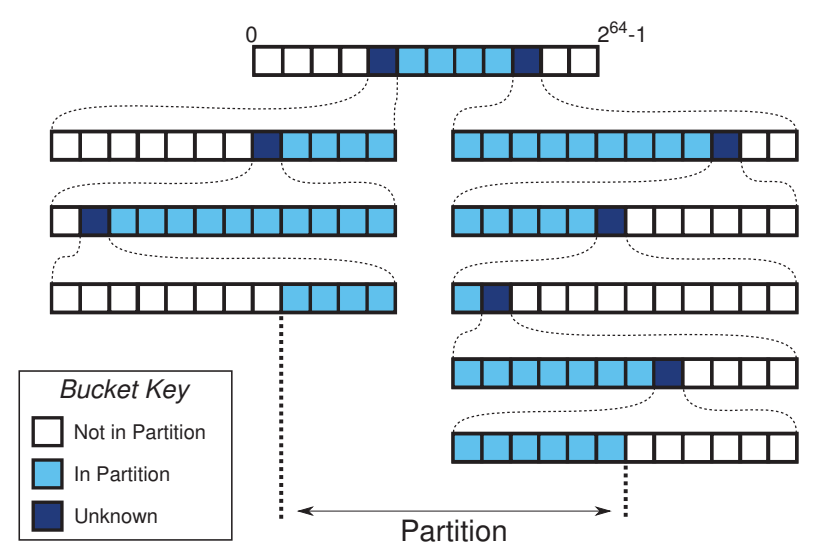

Figure 5: A tablet profile consists of a hierarchical collection of bucket arrays; buckets are subdivided dynamically when their counts become large. The tree structure creates (bounded) uncertainty when assigning partition boundaries, since counts in ancestor buckets may represent objects either before or after the boundary.

counts the number of log records corresponding to a range of object identifiers, along with the total log space consumed by those records. Tablet profiles are updated as new log records are created and old segments are cleaned, and the master periodically scans its tablet profiles to compute a new will.

Unfortunately, it isn't possible to choose the buckets for a tablet profile statically because the space of object identifiers is large $\left(2^{64}\right)$ and clients can allocate object identifiers however they wish. With any static choice of buckets, it is possible that all of the objects in a table could end up in a single bucket, which would provide no information for partitioning. Buckets must be chosen dynamically so that the contents of each bucket are small compared to the contents of a partition.

RAMCloud represents a tablet profile as a dynamic tree of bucket arrays, as shown in Figure 5. Initially the tree consists of a single bucket array that divides the entire 64-bit identifier space into buckets of equal width (in the current implementation there are 256 buckets in each array). Whenever a master creates a new log record it updates the appropriate bucket. If a bucket becomes too large (the number of records or space usage exceeds a threshold) then a child bucket array is created to subdivide the bucket's range into smaller buckets. Future log records are profiled in the child bucket array instead of the parent. However, the counts in the parent bucket remain (RAMCloud does not attempt to redistribute them in the child bucket array since this could require rescanning a large portion of the log). The master decrements bucket counts when it cleans log segments. Each bucket array records the position of the log head when that array was created, and the master uses this information during cleaning to decrement the same bucket that was incremented when the record was created (thus, over time the counts in non-leaf buckets are likely to become small). Bucket arrays are collapsed back into their parents when usage drops.

To calculate partitions, a master scans its tablet profiles in a depth-first search, accumulating counts of records and space usage and establishing partition boundaries whenever the counts reach threshold values. For example, one policy might be to assign partitions based on log space usage so that no partition has more than $600 \mathrm{MB}$ of log data or more than three million objects.

The tablet profile structure creates uncertainty in the actual usage of a partition, as illustrated in Figure 5. If a partition boundary is placed at the beginning of a leaf bucket, it isn't possible to tell whether counts in ancestor buckets belong to the new partition or the previous one. Fortunately, the uncertainty is bounded. For example, in the current RAMCloud implementation, there could be up to 7 ancestor buckets, each of which could account for $8 \mathrm{MB}$ of data (the threshold for subdividing a bucket), for a worst-case uncertainty of $56 \mathrm{MB}$ for each partition boundary. In order to bound recov- 
ery times, RAMCloud pessimistically assumes that unknown counts fall within the current partition.

In the configuration used for RAMCloud, the memory overhead for tablet profiles is $0.6 \%$ in the worst case ( 8 levels of bucket array for $8 \mathrm{MB}$ of data). The parameters of the tablet profile can be changed to make trade-offs between the storage overhead for profiles and the accuracy of partition boundaries.

\subsection{Consistency}

We designed RAMCloud to provide a strong form of consistency (linearizability [13], which requires exactly-once semantics), even across host failures and network partitions. A full discussion of RAMCloud's consistency architecture is beyond the scope of this paper, and the implementation is not yet complete; however, it affects crash recovery in two ways. First, a master that is suspected of failure (a sick master) must stop servicing requests before it can be recovered, to ensure that applications always read and write the latest version of each object. Second, when recovering from suspected coordinator failures, RAMCloud must ensure that only one coordinator can manipulate and serve the cluster's configuration at a time.

RAMCloud will disable a sick master's backup operations when it starts recovery, so the sick master will be forced to contact the coordinator to continue servicing writes. The coordinator contacts backups at the start of recovery to locate a replica of every segment in the sick master's log, including the active segment to which the master may still be writing. Once a backup with a replica of the active segment has been contacted, it will reject backup operations from the sick master with an indication that the master must stop servicing requests until it has contacted the coordinator. Masters will periodically check in with their backups, so disabling a master's backup operations will also stop it from servicing read requests by the time recovery completes.

Coordinator failures will be handled safely using the ZooKeeper service [14]. The coordinator will use ZooKeeper to store its configuration information, which consists of a list of active storage servers along with the tablets they manage. ZooKeeper uses its own replication mechanisms to provide a high level of durability and availability for this information. To handle coordinator failures, the active coordinator and additional standby coordinators will compete for a single coordinator lease in ZooKeeper, which ensures that at most one coordinator runs at a time. If the active coordinator fails or becomes disconnected, its lease will expire and it will stop servicing requests. An arbitrary standby coordinator will acquire the lease, read the configuration information from ZooKeeper, and resume service. The configuration information is small, so we expect to recover from coordinator failures just as quickly as other server failures.

\subsection{Additional Failure Modes}

Our work on RAMCloud so far has focused on recovering the data stored in the DRAM of a single failed master. The sections below describe several other ways in which failures can occur in a RAMCloud cluster and some preliminary ideas for dealing with them; we defer a full treatment of these topics to future work.

\subsubsection{Backup Failures}

RAMCloud handles the failure of a backup server by creating new replicas to replace the ones on the failed backup. Every master is likely to have at least one segment replica on the failed backup, so the coordinator notifies all of the masters in the cluster when it detects a backup failure. Each master checks its segment table to identify segments stored on the failed backup, then it creates new replicas using the approach described in Section 3.2. All of the masters perform their rereplication concurrently and the new replicas are scattered across all of the disks in the cluster, so recovery from backup failures is fast. If each master has 64 GB of memory then each backup will have about 192 GB of data that must be rewritten (assuming 3 replicas for each segment). For comparison, 256 GB of data must be transferred to recover a dead master: 64 GB must be read, then 192 GB must be written during rereplication. 


\begin{tabular}{c|c} 
CPU & Xeon X3470 (4x2.93 GHz cores, 3.6 GHz Turbo) \\
\hline RAM & 16 GB DDR3 at 1333 MHz \\
\hline Disk 1 & WD 2503ABYX (7200 RPM, 250 GB) \\
& Effective read/write: 105/110 MB/s \\
\hline Disk 2 & Seagate ST3500418AS (7200 RPM, 500 GB) \\
& Effective read/write: $108 / 87 \mathrm{MB} / \mathrm{s}$ \\
\hline Flash & Crucial M4 CT128M4SSD2 (128GB) \\
Disks & Effective read/write: 269/182 MB/s \\
\hline NIC & Mellanox ConnectX-2 Infiniband HCA \\
\hline Switches & 5x 36-port Mellanox InfiniScale IV (4X QDR)
\end{tabular}

Table 1: Experimental cluster configuration. All 60 nodes have identical hardware. Effective disk bandwidth is the average throughput from 1,000 $8 \mathrm{MB}$ sequential accesses to random locations in the first $72 \mathrm{~GB}$ of the disk. Flash drives were used in place of disks for Figure 9 only. The cluster has 5 network switches arranged in two layers. Each port's maximum network bandwidth is $32 \mathrm{Gbps}$, but nodes are limited to about 25 Gbps by PCI Express. The switching fabric is oversubscribed, providing at best about $22 \mathrm{Gbps}$ of bisection bandwidth per node when congested.

\subsubsection{Multiple Failures}

Given the large number of servers in a RAMCloud cluster, there will be times when multiple servers fail simultaneously. When this happens, RAMCloud recovers from each failure independently. The only difference in recovery is that some of the primary replicas for each failed server may have been stored on the other failed servers. In this case the recovery masters will use secondary replicas; recovery will complete as long as there is at least one replica available for each segment. It should be possible to recover multiple failures concurrently; for example, if a RAMCloud cluster contains 5,000 servers with flash drives for backup, the measurements in Section 4 indicate that a rack failure that disables 40 masters, each with 64 GB storage, could be recovered in about 2 seconds.

If many servers fail simultaneously, such as in a power failure that disables many racks, RAMCloud may not be able to recover immediately. This problem arises if no replicas are available for a lost segment or if the remaining masters do not have enough spare capacity to take over for all the lost masters. In this case RAMCloud must wait until enough machines have rebooted to provide the necessary data and capacity (alternatively, an operator can request that the system continue with some loss of data). RAMCloud clusters should be configured with enough redundancy and spare capacity to make situations like this rare.

\subsubsection{Cold Start}

RAMCloud must guarantee the durability of its data even if the entire cluster loses power at once. In this case the cluster will need to perform a "cold start" when power returns. Normally, when a backup restarts, it discards all of the segments stored on its disk or flash, since they have already been rereplicated elsewhere. However, in a cold start this information must be preserved. Backups will contact the coordinator as they reboot, and the coordinator will instruct them to retain existing data; it will also retrieve a list of their segments. Once a quorum of backups has become available, the coordinator will begin reconstructing masters. RAMCloud can use the same partitioned approach described above, but it may make more sense to use a different approach where masters are reconstructed exactly as they existed before the cold start. This will be faster than the partitioned approach because masters will not need to write any backup data: the existing backups can continue to serve after the masters are reconstructed.

The current RAMCloud implementation does not perform cold starts.

\section{EVALUATION}

We implemented the RAMCloud architecture described in Sections 2 and 3, and we evaluated the performance and scalability of crash recovery using a 60-node cluster. The cluster hardware consists of standard off-the-shelf components (see Table 1) with the exception of its networking equipment, which is based on Infiniband; with it our end hosts achieve both high bandwidth (25 Gbps) and low 


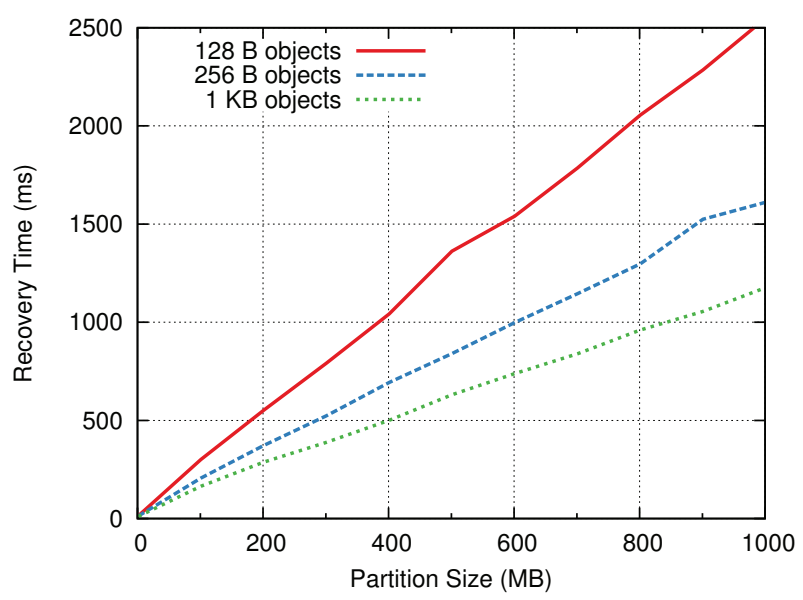

Figure 6: Recovery time as a function of partition size with a single recovery master and 60 backups. Each curve uses objects of a single uniform size.

latency (user-level applications can communicate directly with the NICs to send and receive packets, bypassing the kernel).

The default experimental configuration used one backup server on each machine, with a single disk. A subset of these machines also ran recovery masters. One additional machine ran the coordinator, the crashed master, and the client application. In order to increase the effective scale of the system, some experiments ran two independent backup servers on each machine (each with one disk).

In each experiment a client application observed and measured a crash of a single master and the subsequent recovery. The client initially filled the master with objects of a single size $(1,024$ bytes by default). It then sent a magic RPC to the coordinator which caused it to recover the master. The client waited until all partitions had been successfully recovered, then read a value from one of those partitions and reported the end-to-end recovery time. All experiments used a disk replication factor of 3 (i.e., 3 replicas on disk in addition to one copy in DRAM). The CPUs, disks, and networks were idle and entirely dedicated to recovery (in practice, recovery would have to compete for resources with application workloads, though we would argue for giving priority to recovery).

Each of the subsections below addresses one question related to the performance of recovery. The overall results are:

- A 60-node cluster can recover lost data at about $22 \mathrm{~GB} / \mathrm{sec}$ (a crashed server with $35 \mathrm{~GB}$ storage can be recovered in 1.6 seconds), and recovery performance scales with cluster size. However, our scalability measurements are limited by the small size of our test cluster.

- The speed of an individual recovery master is limited primarily by network speed for writing new segment replicas.

- The segment scattering algorithm distributes segments effectively and compensates for varying disk speeds.

- Fast recovery significantly reduces the risk of data loss.

\subsection{How Large Should Partitions Be?}

Our first measurements provide data for configuring RAMCloud (partition size and number of disks needed per recovery master). Figure 6 measures how quickly a single recovery master can process 


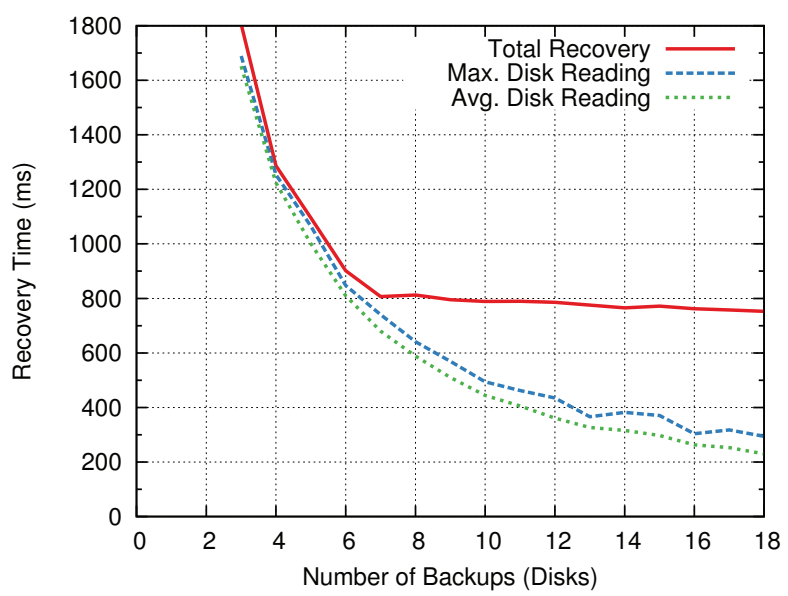

Figure 7: Recovery time as a function of the number of disks, with a single recovery master, one $600 \mathrm{MB}$ partition with 1,024 byte objects, and each disk on a separate machine. "Avg. Disk Reading" measures the average elapsed time (across all disks) to read backup data during recovery; "Max. Disk Reading" graphs the longest time for any disk in the cluster. Once 6-8 disks are available recovery time is limited by the network of the recovery master.

backup data, assuming enough backups to keep the recovery master fully occupied. Depending on the object size, a recovery master can replay log data at a rate of $400-800 \mathrm{MB} / \mathrm{s}$, including the overhead for reading the data from backups and writing new backup copies. With small objects the speed of recovery is limited by the cost of updating the hash table and tablet profiles. With large objects recovery is limited by the network speed during writes to new backups (for example, with $600 \mathrm{MB}$ partitions and a disk replication factor of 3 , the recovery master must write $1.8 \mathrm{~GB}$ of data to backups).

For 1-second recovery Figure 6 suggests that partitions should be limited to no more than $800 \mathrm{MB}$ and no more than 3 million log records (with 128-byte objects a recovery master can process $400 \mathrm{MB}$ of data per second, which is roughly 3 million log records). With $10 \mathrm{Gbps}$ Ethernet, partitions must be limited to $300 \mathrm{MB}$ due to the bandwidth requirements for rereplication.

In our measurements we filled the log with live objects, but the presence of deleted versions will, if anything, make recovery faster. The master's memory has the same log structure as the backup replicas, so the amount of log data to read will always be equal to the size of the master's memory, regardless of deleted versions. However, deleted versions may not need to be rereplicated (depending on the order of replay).

\subsection{How Many Disks Are Needed for Each Recovery Master?}

Each of our disks provided an effective bandwidth of 100-110 MB/s when reading $8 \mathrm{MB}$ segments; combined with Figure 6, this suggests that RAMCloud will need about 6-8 disks for each recovery master in order to keep the pipeline full. Figure 7 graphs recovery performance with one recovery master and a varying number of disks and reaches the same conclusion. With large numbers of disks, the speed of recovery is limited by outbound network bandwidth on the recovery master.

\subsection{How Well Does Recovery Scale?}

The most important issue in recovery for RAMCloud is scalability: if one recovery master can recover $600 \mathrm{MB}$ of data in one second, can 10 recovery masters recover $6 \mathrm{~GB}$ in the same time, and can 100 recovery masters recover $60 \mathrm{~GB}$ ? Unfortunately, the disk bandwidth available in our cluster limited us to 20 recovery masters (120 backups), which is only about $20 \%$ the number we would expect in a fullsize RAMCloud recovery. Nonetheless, within this limited range RAMCloud demonstrates excellent scalability. Figure 8 graphs recovery time as the amount of lost data is increased and the cluster size is increased to match. For each $600 \mathrm{MB}$ partition of lost data, the cluster includes one recovery master 


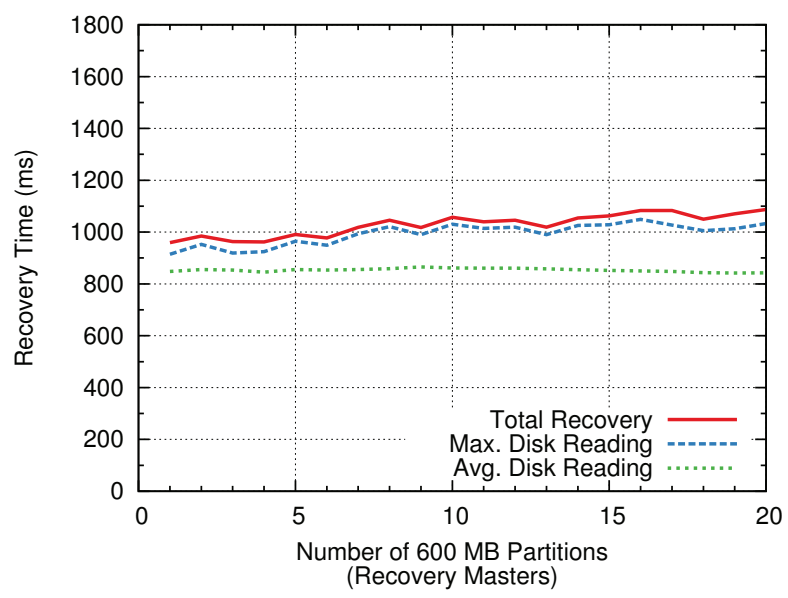

Figure 8: Recovery performance under proportional scaling (one recovery master and 6 backups for each $600 \mathrm{MB}$ partition of data to recover). Each recovery master shared a host with 2 backups, and each point is an average of 5 runs (Figure 11 shows the variance between runs). A horizontal line would indicate perfect scalability. Recovery time is limited by disk bandwidth.

and 6 backups with one disk each. With 20 recovery masters and 120 disks, RAMCloud can recover $11.7 \mathrm{~GB}$ of data in under 1.1 seconds, which is only $13 \%$ longer than it takes to recover $600 \mathrm{MB}$ with a single master and 6 disks.

In order to allow more recovery masters to participate in recovery, we replaced all the disks in our cluster with flash drives, each of which provided $270 \mathrm{MB} / \mathrm{s}$ read bandwidth (as opposed to $110 \mathrm{MB} / \mathrm{s}$ for the disks). With this configuration we were able to run recoveries that used 60 recovery masters, as shown in Figure 9. The system still scales well: with 60 recovery masters RAMCloud can recover $35 \mathrm{~GB}$ of data from a lost server in about 1.6 seconds, which is $26 \%$ longer than it takes 2 recovery masters to recover $1.2 \mathrm{~GB}$ of data.

It is important to keep the overhead for additional masters and backups small, so that recovery can span hundreds of hosts in large clusters. In order to isolate these overheads, we ran additional experiments with artificially small segments $(16 \mathrm{~KB})$ and kept all segment replicas in DRAM to eliminate disk overheads. Figure 10 (bottom curve) shows the recovery time using trivial partitions containing just a single $1 \mathrm{~KB}$ object; this measures the cost for the coordinator to contact all the backups and masters during the setup phase. Our cluster scales to 60 recovery masters with only about a $10 \mathrm{~ms}$ increase in recovery time (thanks in large part to fast RPCs).

Figure 10 also shows recovery time using 1.2 MB partitions and $16 \mathrm{~KB}$ segments (upper curve). In this configuration the cluster performs roughly the same number of RPCs as it does in Figure 8, but it has very little data to process. This exposes the fixed overheads for recovery masters to communicate with backups: as the system scale increases, each master must contact more backups, retrieving less data from each individual backup. Each additional recovery master adds only about $1.5 \mathrm{~ms}$ of overhead, so work can be split across 100 recovery masters without substantially increasing recovery time.

\subsection{How Well Does Segment Scattering Work?}

Figure 11 shows that the segment placement algorithm described in Section 3.2 works well. We measured three different variations of the placement algorithm: the full algorithm, which considers both disk speed and number of segments already present on each backup; a version that uses purely random placement; and an in-between version that attempts to even out the number of segments on each backup but does not consider disk speed. The top graph in Figure 11 shows that the full 


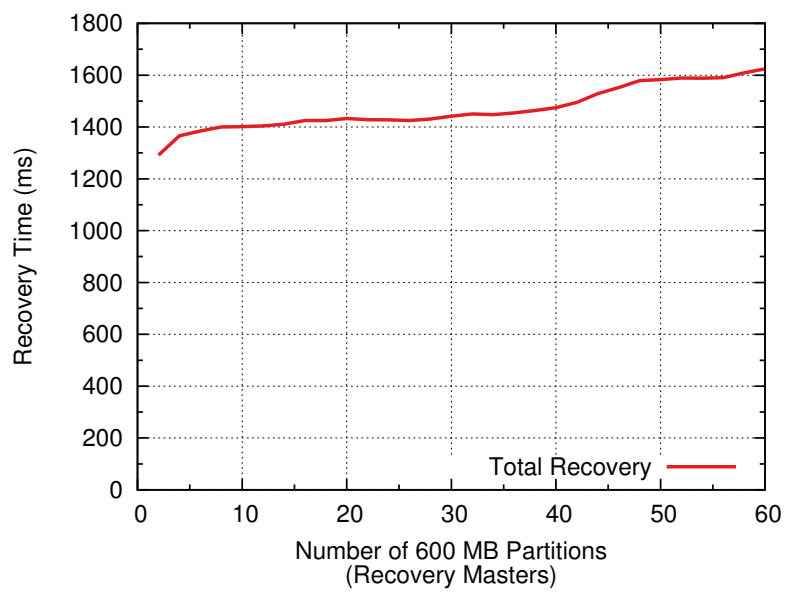

Figure 9: Recovery time under proportional scaling, using flash drives instead of disks. Each partition contained $600 \mathrm{MB}$ of data, and there were 2 backups for each recovery master. As with Figure 8, scaling is proportional: the number of recovery masters and backups increases with the number of partitions being recovered. Each point is an average of 5 runs. A horizontal line would indicate perfect scalability. Recovery is slower than in Figure 8 for a number of reasons: less disk bandwidth available per master $(540 \mathrm{MB} / \mathrm{s}$ vs. $600-660 \mathrm{MB} / \mathrm{s})$, network saturation, and processor and memory contention between the master and backups on each node.

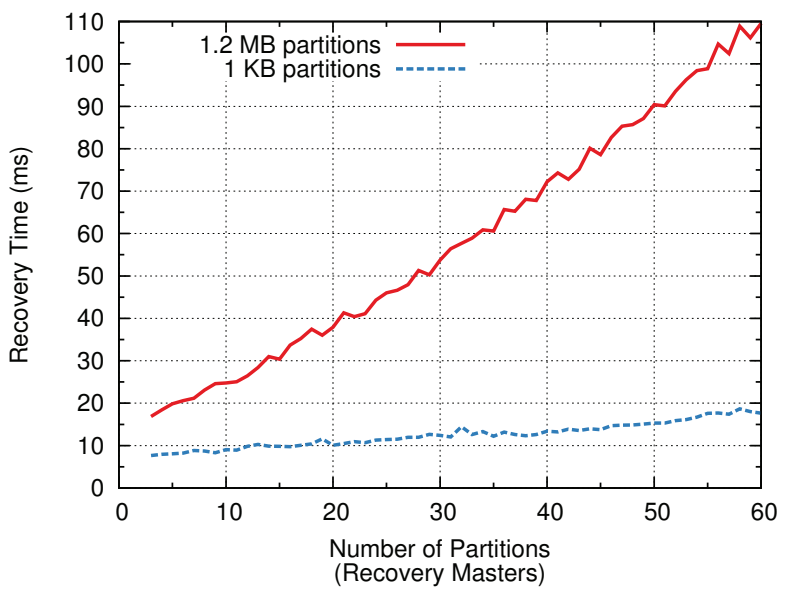

Figure 10: Management overhead as a function of system scale. Partition size is reduced to $16 \mathrm{~KB}$ and segment replicas are stored in DRAM in order to eliminate overheads related to data size or disk. For "1 KB partitions" each partition only contains a single object; this measures the coordinator's overheads for contacting masters and backups. "1.2 MB partitions" maintains the same number of segments (and roughly the same number of RPCs) as in Figure 8; it measures the overhead for masters to contact more and more backups as cluster size increases. Each data point is the average over 5 runs, and there were 2 backups for each recovery master. 

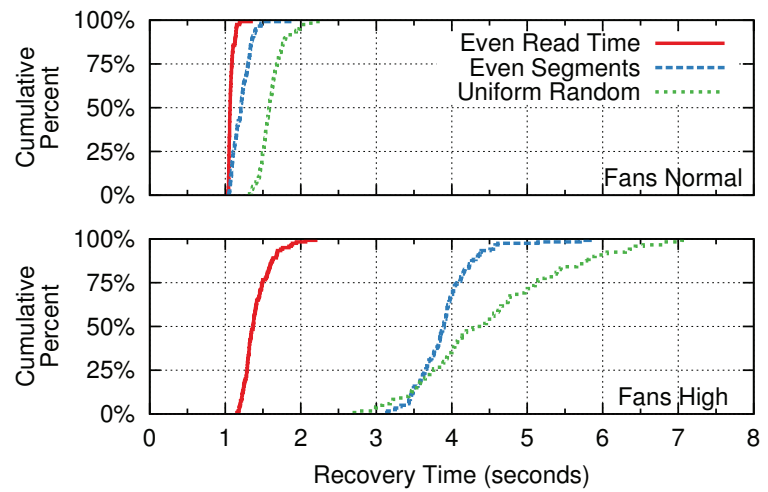

Figure 11: Impact of segment placement on recovery time. Each line is a cumulative distribution of 120 recoveries of twenty $600 \mathrm{MB}$ partitions, showing the percent of recoveries that completed within a given time. "Even Read Time" uses the placement algorithm described in Section 3.2; "Uniform Random" uses a purely random approach; and "Even Segments" attempts to spread segments evenly across backups without considering disk speed. The top graph measured the cluster in its normal configuration, with relatively uniform disk performance; the bottom graph measured the system as it was shipped (unnecessarily high fan speed caused vibrations that degraded performance significantly for some disks). With fans at normal speed, "Even Read Time" and "Even Segments" perform nearly the same since there is little variation in disk speed.

algorithm improves recovery time by about $33 \%$ over a purely random placement mechanism. Much of the improvement came from evening out the number of segments on each backup; considering disk speed improves recovery time by only $12 \%$ over the even-segment approach because the disks did not vary much in speed.

To further test how the algorithm handles variations in disk speed, we also took measurements using the configuration of our cluster when it first arrived. The fans were shipped in a "max speed" debugging setting, and the resulting vibration caused large variations in speed among the disks (as much as a factor of $4 \mathrm{x}$ ). In this environment the full algorithm provided an even larger benefit over purely random placement, but there was relatively little benefit from considering segment counts without also considering disk speed (Figure 11, bottom graph). RAMCloud's placement algorithm compensates effectively for variations in the speed of disks, allowing recovery times almost as fast with highly variable disks as with uniform disks. Disk speed variations may not be significant in our current cluster, but we think they will be important in large datacenters where there are likely to be different generations of hardware.

\subsection{Will Scattering Result in Data Loss?}

RAMCloud's approach of scattering segment replicas allows faster recovery, but it increases the system's vulnerability in the event of simultaneous node failures. For example, consider a cluster with 1,000 nodes and 2x disk replication. With RAMCloud's scattering approach to segment placement, there is a 5\% chance that data will be lost if any 3 nodes fail simultaneously (the three nodes will account for the master and both backups for at least one segment). In contrast, if each master concentrates all its segment replicas on two backups, as in Figure 3a, the probability of data loss drops to less than $10^{-5}$ with 3 simultaneous failures.

Fortunately, the fast recovery enabled by scattering makes it unlikely that a second or third failure will occur before a first failure has been recovered, and this more than makes up for the additional vulnerability, as shown in Figure 12. With one-second recovery the probability of data loss is very low (about $10^{-5}$ in one year even with a 100,000-node cluster). The risk of data loss rises rapidly with recovery time: if recovery takes 1,000 seconds, then RAMCloud is likely to lose data in any one-year period. The line labeled "100s" corresponds roughly to the recovery mechanisms in other systems such as GFS and HDFS (these systems keep 3 replicas on disk, vs. 1 replica in DRAM and 2 


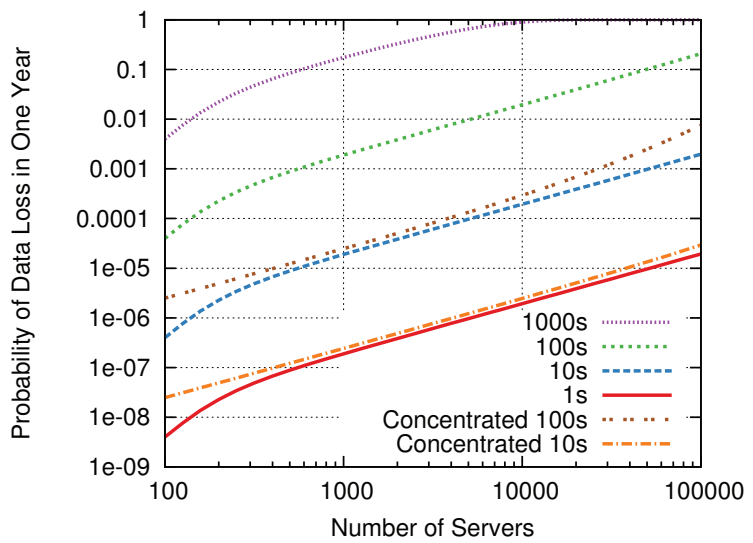

Figure 12: Probability of data loss in one year as a function of cluster size, assuming 8,000 segments per master, two disk replicas for each DRAM copy, and two crashes per year per server with a Poisson arrival distribution. Different lines represent different recovery times. Lines labeled "Concentrated" assume that segments are concentrated instead of scattered: each master picks 2 backups at random and replicates all of its segments on each of those backups.

replicas on disk for the corresponding RAMCloud); with large cluster sizes these other systems may be vulnerable to data loss. Using a concentrated approach rather than scattering improves reliability, but the benefit from faster recovery is much larger: a 10x improvement in recovery time improves reliability more than a 1,000x reduction in scattering.

One risk with Figure 12 is that it assumes server failures are independent. There is considerable evidence that this is not the case in datacenters [23, 8]; for example, it is not unusual for entire racks to become inaccessible at once. Thus it is important for the segment scattering algorithm to consider sources of correlated failure, such as rack boundaries. If there are unpredictable sources of correlated failure, they will result in longer periods of unavailability while RAMCloud waits for one or more of the backups to reboot (RAMCloud is no better or worse than other systems in this respect).

Although we made all of the performance measurements in this section with $3 \mathrm{x}$ disk replication to be conservative, Figure 12 suggests that the combination of two copies on disk and one copy in DRAM should be quite safe. The main argument for 3x disk replication is to ensure 3-way redundancy even in the event of a datacenter power outage, which would eliminate the DRAM copies. With 3x disk replication in addition to the DRAM copy, the likelihood of data loss is extremely small: less than $1 \%$ in a year even with 100,000 servers and 1,000 -second recovery times.

\subsection{What Is the Fastest Possible Recovery?}

Assuming that recovery is scalable, it should be possible to recover even faster than 1-2 seconds by using more backups and more recovery masters, with smaller partitions. However, we think that it will be difficult to recover faster than a few hundred milliseconds without significant changes to the recovery mechanism. For example, RAMCloud currently requires 150 milliseconds just to detect failure, and the time for the coordinator to contact every backup may approach $100 \mathrm{~ms}$ in a large cluster. In addition, it takes nearly $100 \mathrm{~ms}$ to read a single segment from disk (but this could be reduced if flash memory replaces disk for backup storage).

\section{RISKS}

There are three risks associated with RAMCloud's recovery mechanism that we have not been able to fully evaluate yet. We hope to learn more about these risks (and devise solutions, if necessary) as we gain more experience with the system. 
Scalability. The measurements of scalability in Section 4.3 are encouraging, but they are based on a cluster size about one-fifth of what we would expect in production. It seems likely that larger clusters will expose problems that we have not yet seen.

Over-hasty recovery. In order to recover quickly, RAMCloud must also detect failures quickly. Whereas traditional systems may take 30 seconds or more to decide that a server has failed, RAMCloud makes that decision in $150 \mathrm{~ms}$. This introduces a risk that RAMCloud will treat performance glitches as failures, resulting in unnecessary recoveries that could threaten both the performance and the integrity of the system. Furthermore, fast failure detection precludes some network protocols. For example, most TCP implementations wait $200 \mathrm{~ms}$ before retransmitting lost packets; if TCP is to be used in RAMCloud, either its retransmit interval must be shortened or RAMCloud's failure detection interval must be lengthened. The current implementation of RAMCloud supports several transport protocols for its RPC system (including TCP), most of which support fast failure detection.

Fragmented partitions. Our approach to recovery assumes that a master's objects can be divided into partitions during recovery. However, this changes the locality of access to those objects, which could degrade application performance after recovery. Our current data model does not benefit much from locality, but as we experiment with richer data models, this issue could become important.

\section{RELATED WORK}

There are numerous examples where DRAM has been used to improve the performance of storage systems. Early experiments in the 1980s and 1990s included file caching [19] and main-memory database systems $[10,11]$. In recent years, large-scale Web applications have found DRAM indispensable to meet their performance goals. For example, both Google and Yahoo! keep their entire Web search indexes in DRAM; Facebook offloads its database servers by caching tens of terabytes of data in DRAM with memcached [3]; and Bigtable allows entire column families to be loaded into memory [6]. RAMCloud differs from these systems because it keeps all data permanently in DRAM (unlike Bigtable and Facebook, which use memory as a cache on a much larger disk-based storage system) and it is general-purpose (unlike the Web search indexes).

There has recently been a resurgence of interest in main-memory databases. One example is $\mathrm{H}$ Store [16], which keeps all data in DRAM, supports multiple servers, and is general-purpose. However, H-Store is focused more on achieving full RDBMS semantics and less on achieving large scale or low latency to the same degree as RAMCloud. H-Store keeps redundant data in DRAM and does not attempt to survive coordinated power failures.

A variety of "NoSQL" storage systems have appeared recently, driven by the demands of large-scale Web applications and the inability of relational databases to meet their needs. Examples include Dynamo [9] and PNUTS [7]. Many of these systems use DRAM in some form, but all are fundamentally disk-based and none are attempting to provide latencies in the same range as RAMCloud. These systems provide availability using symmetric replication instead of fast crash recovery.

RAMCloud is similar in many ways to Google's Bigtable [6] and GFS [12]. Bigtable, like RAMCloud, implements fast crash recovery (during which data is unavailable) rather than online replication. Bigtable also uses a log-structured approach for its (meta)data, and it buffers newly-written data in memory, so that write operations complete before data has been written to disk. GFS serves a role for Bigtable somewhat like the backups in RAMCloud. Both Bigtable and GFS use aggressive data partitioning to speed up recovery. However, Bigtable and GFS were designed primarily for disk-based datasets; this allows them to store 10-100x more data than RAMCloud, but their access latencies are 10-100x slower (even for data cached in DRAM).

Caching mechanisms such as memcached [3] appear to offer a particularly simple mechanism for crash recovery: if a caching server crashes, its cache can simply be re-created as needed, either on the crashed server (after it restarts) or elsewhere. However, in large-scale systems, caching approaches 
can cause large gaps in availability after crashes. Typically these systems depend on high cache hit rates to meet their performance requirements; if caches are flushed, the system may perform so poorly that it is essentially unusable until the cache has refilled. This happened in an outage at Facebook in September 2010 [1]: a software error caused 28 TB of memcached data to be flushed, rendering the site unusable for 2.5 hours while the caches refilled from slower database servers.

Randomization has been used by several previous systems to allow system management decisions to be made in a distributed and scalable fashion. For example, consistent hashing uses randomization to distribute objects among a group of servers [24, 9]. Mitzenmacher and others have studied the theoretical properties of randomization with refinement and have shown that it produces near-optimal results $[17,5]$.

RAMCloud's log-structured approach to storage management is similar in many ways to log-structured file systems (LFS) [21]. However, log management in RAMCloud is simpler and more efficient than in LFS. RAMCloud is simpler because the log need not contain metadata to enable random-access reads as in LFS: the hash table enables fast access to data in DRAM, and the disk log is never read except during recovery, at which time the entire log is read. Thus the log consists primarily of object records and tombstones that mark their deletion. RAMCloud does not require checkpoints as in LFS, because it replays the entire log during recovery. RAMCloud is more efficient than LFS because it need not read data from disk during cleaning: all live data is always in memory. The only I/O during cleaning is to rewrite live data at the head of the log; as a result, RAMCloud consumes 3-10x less bandwidth for cleaning than LFS (cleaning cost has been a controversial topic for LFS; see [22], for example).

\section{CONCLUSION}

In this paper we have demonstrated that the resources of a large-scale storage system can be used to recover quickly from server crashes. RAMCloud distributes backup data across a large number of secondary storage devices and employs both data parallelism and pipelining to achieve end-to-end recovery times of 1-2 seconds. Although we have only been able to evaluate RAMCloud on a small cluster, our measurements indicate that the techniques will scale to larger clusters. Our implementation uses a simple log-structured representation for data, both in memory and on secondary storage, which provides high write throughput in addition to enabling fast recovery.

Fast crash recovery is a key enabler for RAMCloud: it allows a high-performance DRAM-based storage system to provide durability and availability at one-third the cost of a traditional approach using online replicas.

\section{ACKNOWLEDGMENTS}

Asaf Cidon reeducated us on the fundamentals of probability and assisted us with several calculations, including Figure 12. Nanda Kumar Jayakumar helped us with performance measurements and some of the figures in the paper. Several people provided helpful feedback on the paper, including Asaf Cidon, Ankita Kejriwal, Kay Ousterhout, George Varghese, the anonymous SOSP reviewers, and our shepherd Geoff Voelker. This work was supported by the Gigascale Systems Research Center and the Multiscale Systems Center, two of six research centers funded under the Focus Center Research Program, a Semiconductor Research Corporation program, and by Facebook, Mellanox, NEC, NetApp, SAP, and Samsung. This work was also partially supported by NSF Cybertrust awards CNS-0716806 and CNS-1052985 (CT-T: A Clean-Slate Infrastructure for Information Flow Control). Diego Ongaro is supported by The Junglee Corporation Stanford Graduate Fellowship. Steve Rumble is supported by a Natural Sciences and Engineering Research Council of Canada Postgraduate Scholarship.

\section{REFERENCES}

[1] More Details on Today's Outage | Facebook, Sept. 2010.

http: //www. facebook. com/note.php?note_id=431441338919.

[2] Agiga tech agigaram, Mar. 2011. http://www.agigatech.com/agigaram.php. 
[3] memcached: a distributed memory object caching system, Jan. 2011.

http: //www. memcached.org/.

[4] M. K. Aguilera, A. Merchant, M. Shah, A. Veitch, and C. Karamanolis. Sinfonia: A new paradigm for building scalable distributed systems. ACM Trans. Comput. Syst., 27:5:1-5:48, November 2009.

[5] Y. Azar, A. Z. Broder, A. R. Karlin, and E. Upfal. Balanced allocations (extended abstract). In Proceedings of the twenty-sixth annual ACM symposium on theory of computing, STOC '94, pages 593-602, New York, NY, USA, 1994. ACM.

[6] F. Chang, J. Dean, S. Ghemawat, W. C. Hsieh, D. A. Wallach, M. Burrows, T. Chandra, A. Fikes, and R. E. Gruber. Bigtable: A distributed storage system for structured data. ACM Trans. Comput. Syst., 26:4:1-4:26, June 2008.

[7] B. F. Cooper, R. Ramakrishnan, U. Srivastava, A. Silberstein, P. Bohannon, H.-A. Jacobsen, N. Puz, D. Weaver, and R. Yerneni. Pnuts: Yahoo!'s hosted data serving platform. Proc. VLDB Endow., 1:1277-1288, August 2008.

[8] J. Dean. Keynote talk: Evolution and future directions of large-scale storage and computation systems at google. In Proceedings of the 1st ACM symposium on Cloud computing, Jun 2010.

[9] G. DeCandia, D. Hastorun, M. Jampani, G. Kakulapati, A. Lakshman, A. Pilchin,

S. Sivasubramanian, P. Vosshall, and W. Vogels. Dynamo: amazon's highly available key-value store. In Proceedings of twenty-first ACM SIGOPS symposium on operating systems principles, SOSP '07, pages 205-220, New York, NY, USA, 2007. ACM.

[10] D. J. DeWitt, R. H. Katz, F. Olken, L. D. Shapiro, M. R. Stonebraker, and D. A. Wood. Implementation techniques for main memory database systems. In Proceedings of the 1984 ACM SIGMOD international conference on management of data, SIGMOD '84, pages 1-8, New York, NY, USA, 1984. ACM.

[11] H. Garcia-Molina and K. Salem. Main memory database systems: An overview. IEEE Trans. on Knowl. and Data Eng., 4:509-516, December 1992.

[12] S. Ghemawat, H. Gobioff, and S.-T. Leung. The google file system. In Proceedings of the nineteenth ACM symposium on Operating systems principles, SOSP '03, pages 29-43, New York, NY, USA, 2003. ACM.

[13] M. P. Herlihy and J. M. Wing. Linearizability: a correctness condition for concurrent objects. ACM Trans. Program. Lang. Syst., 12:463-492, July 1990.

[14] P. Hunt, M. Konar, F. P. Junqueira, and B. Reed. Zookeeper: wait-free coordination for internet-scale systems. In Proceedings of the 2010 USENIX annual technical conference, USENIX ATC'10, pages 11-11, Berkeley, CA, USA, 2010. USENIX Association.

[15] R. Johnson and J. Rothschild. Personal Communications, March 24 and August 20, 2009.

[16] R. Kallman, H. Kimura, J. Natkins, A. Pavlo, A. Rasin, S. Zdonik, E. P. C. Jones, S. Madden, M. Stonebraker, Y. Zhang, J. Hugg, and D. J. Abadi. H-store: a high-performance, distributed main memory transaction processing system. Proc. VLDB Endow., 1:1496-1499, August 2008.

[17] M. D. Mitzenmacher. The power of two choices in randomized load balancing. $\mathrm{PhD}$ thesis, University of California, Berkeley, 1996. AAI9723118.

[18] J. Ousterhout, P. Agrawal, D. Erickson, C. Kozyrakis, J. Leverich, D. Mazières, S. Mitra, A. Narayanan, D. Ongaro, G. Parulkar, M. Rosenblum, S. M. Rumble, E. Stratmann, and R. Stutsman. The case for ramcloud. Commun. ACM, 54:121-130, July 2011.

[19] J. K. Ousterhout, A. R. Cherenson, F. Douglis, M. N. Nelson, and B. B. Welch. The sprite network operating system. Computer, 21:23-36, February 1988.

[20] D. A. Patterson, G. Gibson, and R. H. Katz. A case for redundant arrays of inexpensive disks (raid). In Proceedings of the 1988 ACM SIGMOD international conference on management of data, SIGMOD '88, pages 109-116, New York, NY, USA, 1988. ACM.

[21] M. Rosenblum and J. K. Ousterhout. The design and implementation of a log-structured file system. ACM Trans. Comput. Syst., 10:26-52, February 1992.

[22] M. Seltzer, K. A. Smith, H. Balakrishnan, J. Chang, S. McMains, and V. Padmanabhan. File system logging versus clustering: a performance comparison. In Proceedings of the USENIX 1995 Technical Conference, TCON'95, pages 21-21, Berkeley, CA, USA, 1995. USENIX 


\section{Association.}

[23] K. Shvachko, H. Kuang, S. Radia, and R. Chansler. The hadoop distributed file system. In Proceedings of the 2010 IEEE 26th Symposium on Mass Storage Systems and Technologies (MSST), MSST '10, pages 1-10, Washington, DC, USA, 2010. IEEE Computer Society.

[24] I. Stoica, R. Morris, D. Liben-Nowell, D. R. Karger, M. F. Kaashoek, F. Dabek, and H. Balakrishnan. Chord: a scalable peer-to-peer lookup protocol for internet applications. IEEE/ACM Trans. Netw., 11:17-32, February 2003. 